\title{
Simulation of automatic control optimization model based on improved PID algorithm
}

\author{
Liu yong \\ (Department of electrical engineering Shandong Polytechnic，Jinan Shandong 250104,China)
}

Keywords: improved PID algorithm; automatic control; stability;

\begin{abstract}
: the stable control for industrialization automatic control system is the key for stable working of automatic control system. In the process of automatic control with the traditional algorithm, the influence of the outside interference factor to the automatic control system is not considered, thus, the stability of the automatic control system is reduced. An automatic control method based on improved PID algorithm is proposed. The control principle of the PID algorithm is analyzed, the controlled quantity is regarded as variables, using neural network algorithm to optimize PID algorithm, through training and learning of neural network to change the stability of PID control algorithm. The simulation results show that the improved algorithm can improve the stability of the automatic control system, and the effect is satisfactory.
\end{abstract}

\section{Introduction}

With the continuous improvement of the level of industrialization, the scale of automatic control system in industrial production is getting bigger, operation process becomes more and more complex, the existence of various nonlinear disturbance signal, resulting in poor running stability of control system [1,2]. Therefore, how to control the stability of the automatic control system has become a hot research topic in the field of industrial control [3].

At present, the main automatic control method mainly includes the method based on adaptive fuzzy control, based on the PID algorithm and the neural network [4-6]. The most commonly used is the automatic control method based on neural network. Due to the automatic control method has an irreplaceable role in the field of industrial control, therefore, has broad prospects for development, and become the focus of research [7]. But the traditional automatic control method has no consideration for outside interference factor, and has the low stability of the automatic control system [8-10].

To this, an automatic control method based on improved PID algorithm is proposed. The control principle of the PID algorithm is analyzed, the controlled quantity is regarded as variables, using neural network algorithm to optimize PID algorithm, through training and learning of neural network to change the stability of PID control algorithm. The simulation results show the superiority of the improved algorithm in the automatic control.

\section{2 the principle of automatic control method based on improved PID}

In the process of automatic control with the traditional algorithm, the influence of the outside interference factor to the automatic control system is not considered, thus, the stability of the automatic control system is reduced. An automatic control method based on improved PID algorithm is proposed.

\section{1 establishment of improved PID control model}

The control algorithm of the traditional PID is as follows:

$$
\begin{aligned}
& u(k)=u(k-1)+K_{p}[e(k)-e(k-1)] \\
& +K_{i}[e(k)]+k_{d}[e(k)-2 e(k-1)+e(k-2)]
\end{aligned}
$$

If the $\mathrm{K}_{\mathrm{p}}, \mathrm{K}_{\mathrm{d}}, \mathrm{K}_{\mathrm{i}}$ are viewed as adjustable coefficient depending on the operating state of the system, equation (1) can be described as: 
$u(k)=f\left[u(k-1), K_{p}, K_{i}, K_{d}, e(k), e(k-1), e(k-2)\right]$

In the upper formula, $f[\bullet]$ is a nonlinear function associated with $K_{p}, K_{d}, K_{i}, u(k-1)$, e (k), and such an optimal control law can be found by BP neural network through training and learning. PID controller of BP neural network is composed of two parts: 1) the classical PID controller: closed-loop control for the controlled object directly; 2) neural network according to the opreating state of the system, online tuning three adjustable parameters $\mathrm{K}_{\mathrm{p}}, \mathrm{K}_{\mathrm{i}}, \mathrm{K}_{\mathrm{d}}$ of PID controller, in order to find the optimal control laws.

In the improved algorithm, BP neural network with simple structure of three layers BP neural network, $\mathrm{M}$ input nodes, $\mathrm{Q}$ hidden layer nodes and three output nodes, the input of the network input layer is: $x_{i} \quad i=1,2, \cdots M$, the number of input variables $M$ is depending on the complexity of automatic control system.

The input and output of the network hidden layer are respectively:

$n e t_{j}(k)=\sum_{i} w_{j i} x_{i}, y_{j}(k)=f\left(\sum_{i} w_{j i} x_{i}\right)=f\left(\right.$ net $\left._{j}(k)\right), j=1,2, \cdots Q$

The input

and output of the output layer are respectively:

$\operatorname{net}_{l}(k)=\sum_{j} v_{l j} y_{j}, z_{l}(k)=f\left(\sum_{j} v_{l j} y_{j}\right)=f\left(\right.$ net $\left._{l}(k)\right) \quad, \quad l=1,2,3$

From the BP neural network principle, the three parameters of the PID controller are:

$K_{p}(k)=z_{1}(k), K_{i}(k)=z_{2}(k), K_{d}(k)=z_{3}(k)$

Taking the performance index function as:

$E(k)=\frac{1}{2}[r(k)-q(k)]^{2}=\frac{1}{2} e^{2}(k)$

According to the steepest descent method, the weight coefficient of the network is adjusted, that is, based on $\mathrm{E}$ to search and adjust the negative gradient direction of the weight coefficient, and the inertia term of searching the global minimum of fast convergence is added:

$\Delta v_{l j}(k)=-\eta \frac{\partial E(k)}{\partial v_{l j}}+\alpha \Delta v_{l j}(k-1)$

In the formula, $\eta$ is the learning rate and $\alpha$ is the smoothing factor.

$\frac{\partial E(k)}{\partial v_{l j}}=\frac{\partial E(k)}{\partial q(k)} \cdot \frac{\partial q(k)}{\partial u(k)} \cdot \frac{\partial u(k)}{\partial z_{l}(k)} \cdot \frac{\partial z_{l}(k)}{\partial n e t_{1}(k)} \cdot \frac{\partial n e t_{l}(k)}{\partial v_{l j}}$

$\frac{\partial \operatorname{net}_{l}(k)}{\partial v_{l j}}=y_{j}(k)$

Because of the unknown $\frac{\partial q(k)}{\partial u(k)}$, the approximation is replaced by symbol function $\operatorname{sgn}\left[\frac{\partial q(k)}{\partial u(k)}\right]$, and the resulting influence of imprecise control can be compensated by adjusting learning speed $\eta$.

From equation (8) and (9) can be obtained

$\frac{\partial u(k)}{\partial z_{1}(k)}=e(k)-e(k-1), \frac{\partial u(k)}{\partial z_{2}(k)}=e(k), \frac{\partial u(k)}{\partial z_{3}(k)}=e(k)-2 e(k-1)+e(k-2)$

Therefore, the weight coefficient adjust equation of the output layer of the BP neural network is.:

$\Delta v_{l j}(k)=\eta \delta_{l} y_{j}(k)+\alpha \Delta v_{l j}(k-1)$

$\delta_{l}=e(k) \cdot \operatorname{sgn}\left[\frac{\partial q(k)}{\partial u(k)}\right] \cdot \frac{\partial u(k)}{\partial z_{l}(k)} \cdot g^{\prime}\left[n e t_{l}(k)\right]$

According to the above derivation method, the weight coefficient adjust equation of the hidden layer is: 


$$
\begin{aligned}
& \Delta w_{j i}(k)=\eta \delta_{j} x_{i}(k)+\alpha \Delta w_{j i}(k-1) \\
& \delta_{j}=f^{\prime}\left[\text { net }_{j}(k)\right] \cdot \sum_{l=1}^{3} \delta_{l} v_{l j}(k)
\end{aligned}
$$

in the formula, $g^{\prime}[\bullet]=g(x)[1-g(x)], \quad f^{\prime}[\bullet]=\left[1-f^{2}(x)\right] / 2$.

According to the method described above, the traditional PID algorithm is analyzed, training and learning PID controlled quantity with neural network, through the establishment of automatic control optimization model, finally realizes the smooth control of automatic control system.

\section{2 automatic control implementation process}

The process of automatic control implementation based on the improved PID algorithm is described as follows:

1) the structure of BP neural network is selected, that is, the number of nodes $M$ of hidden layer and the number of node $\mathrm{Q}$ of the input layer are selected;

i2) $r(k)$ and $q(k)$ are obtained by sampling, and the $e(k)=r(k)-q(k)$ is calculated.;

3) e(i), r(i), q(i) and $\mathrm{u}(\mathrm{i}-1)(\mathrm{i}=\mathrm{k}, \mathrm{k}-1, \cdots, \mathrm{k}-\mathrm{p})$ are normalized as the input of neural network;

4) calculate the input and output of the neurons in the each layer of BP neural network, the output of output layer of the BP neural network is the three adjustable parameters $K_{i}, K_{d}$, $K_{p}$ of the PID controller,;

5) Controlled output $\mathrm{u}(\mathrm{k})$ of PID controller is calculated, which is involved in the closed-loop control and calculation;

6) The error is calculated according to the performance index function (13), and if the error meets the request, end, otherwise continue;

7) calculate the weight coefficient $\mathrm{v}_{\mathrm{lj}}(\mathrm{k}), \mathrm{w}_{\mathrm{ji}}(\mathrm{k})$ of output layer and hidden layer;

8) Set $k=k+1$, return the loop.

According to the method described above, the controlled quantity is regarded as variables, using neural network algorithm to optimize PID algorithm, through training and learning of neural network to change the stability of PID control algorithm.

\section{3 experimental results and analysis}

\section{1 experimental environment setting}

Research object is automatic control system of one city water plant, the rated pressure of the flow is $1.25 \mathrm{MPa}$, rated flow is $6 t / \mathrm{h}$. Simulink is used to simulate flow pressure model based on the fuzzy controller, and compared with the fuzzy PID control model.

Establishing the mathematical model of the controlled object. The transfer function of the flow pressure in each stage is as follows: the boost stage is $G_{1}(s)=0.05 e^{-20 s} /(60 s+1)$, the conveying section is $G_{2}(s)=0.8 /(100 s+1)$, the circulating system flow section is $G_{3}(s)=0.5\left(1-e^{-100 s}\right) / 100 s$, the buck section is $G_{4}(s)=1.5\left(1-e^{-80 s}\right) / 80 s$.

\section{2 experimental results contrast analysis}

When the system does not add the disturbance, namely, when Step2 value is 0, the simulation curve of flow pressure adjustment system is shown in Figure 1. Available from the diagram, when it is under PID control. The system has large overshoot, time for reaching the steady state is 3100s; under improved PID control, the overshoot of the system is only $4.23 \%$, time for reaching steady time is 1000s. It can be seen that the use of improved PID can get smaller overshoot and shorter stability time than the traditional PID controller. 


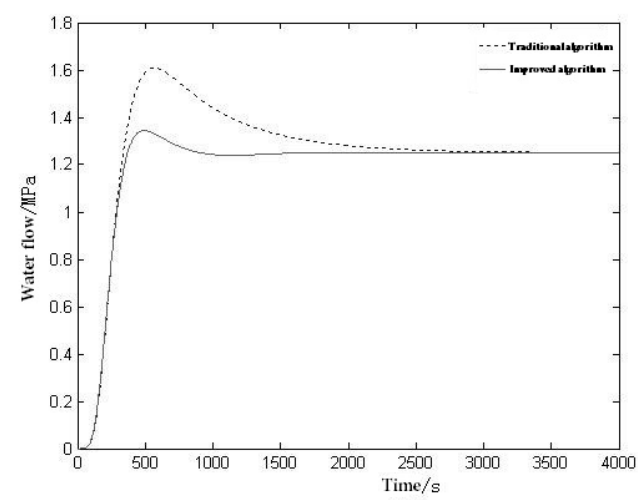

Fig. 1 simulation curve of water flow pressure regulation without disturbance

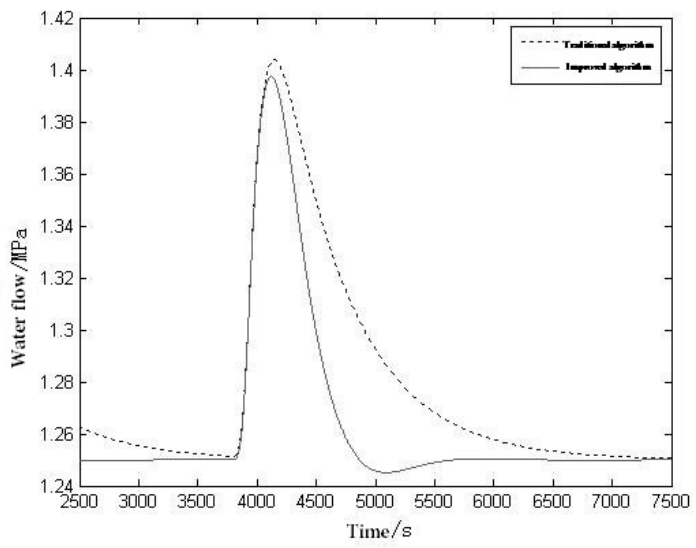

Figure 2 adjusted simulation curve when $T_{31}=120 \mathrm{~s}, T_{32}=70 \mathrm{~s}$

In the control of water, there are many factors influencing the flow pressure, the characteristics of the system is not invariable, and bound to some changes due to various reasons, therefore, time constant for circulating water system $T_{31}=100 \mathrm{~s}$, boost time constant $T_{32}=80 \mathrm{~s}$ and time constant for circulating water system $T_{31}=120 \mathrm{~s}$, boost time constant $T_{32}=70 \mathrm{~s}$, two cases were simulated as shown in Figure 2. By comparison, it can be seen that adaptability of the controlled object parameters change of improved PID control is obviously superior to the traditional PID control, and has better robustness.

\section{Conclusion}

Aiming at the defects of the traditional algorithm, an automatic control method based on improved PID algorithm is proposed. The control principle of the PID algorithm is analyzed, the controlled quantity is regarded as variables, using neural network algorithm to optimize PID algorithm, through training and learning of neural network to change the stability of PID control algorithm. The simulation results show that the improved algorithm can improve the stability of the automatic control system, and the effect is satisfactory.

\section{References:}

[1] Yang Qiyao, Zhou Kongkang, Zhang Wenna, et al. Fuzzy-PID Control on Semi-active Air Suspension [J]. Transactions of the Chinese society for agricultural machinery, 2008, 39 (9): 24-29.

[2] Yang Wenchao, Zhang Rongbiao, Zhang Chunyan. The Research of Realizing PID Controler Based on FPGA [J]. Microcomputer information, 2005, (32): 135-137.

[3] Sun Zongyi, Wang Bailin. The Study and Simulation about A Kind Of Improved Fuzzy-PID Controller [J]. Computer simulation, 2003, 20 (2): 79-81.

[4] Li Sumin, Wang Jie, Song Beiguang. An improved PID control algorithm [J]. Microcomputer information, 2006, 22 (25): 40-41. 
[5] Gao Binna. The applications of digital PID control for motion control system [J]. Journal of China Institute of Electronic Science, 2006, 1 (6): 564-567.

[6] Wu Changjun. The improvement of PID fuzzy-based controller [J]. Journal of Anhui University of Technology and Science (NATURAL SCIENCE EDITION), 2004, (1): 40-43.

[7] Liu Bin, Tan Jianping, Huang Changzheng. Research and Application of an Improved PID Control Algorithm [J]. Micro computer information, 2007, 23 (16): 15-17.

[8] Li Guoyong. An improved adaptive PID controller [J]. Journal of Taiyuan University of Technology, 2003, 34 (1): 51-52.

[9] Wang Jin, Shang Yulin, Li Jing. The application of fuzzy adaptive PID controller in the molybdenum wire constant tension control of wire-cutting machine [J]. Journal of Xi'an College of Engineering Science and Technology, 19, 2005 (4): 460-462.

[10] Wang Chunling, Dong Bin. Improving the PID-control Using Digital Filter [J]. Instrumentation technology, 2002, (3): 32-33. 